\title{
Factors Associated with Falls in Psychogeriatric Inpatients and Comparison of Two Fall Risk Assessment Tools
}

\author{
MMC Wong, PF Pang
}

\begin{abstract}
Objectives: Development of a fall prevention programme for psychogeriatric inpatients is required. Therefore, the present study aimed to investigate factors associated with falls in psychogeriatric inpatients and compare two fall risk assessment tools.

Methods: This study had two parts. First, all fall reports involving psychogeriatric inpatients in United Christian Hospital in 2016 were retrospectively reviewed. Factors associated with the falls were identified by comparing patients who had a fall incident and those who did not. Second, in a pilot study, 30 consecutive psychogeriatric inpatients were assessed for fall risk using the Morse Fall Scale (MFS) and the Wilson Sims Fall Risk Assessment Tool (WSFRAT), and outcomes were then compared with the modified Functional Ambulation Classification (MFAC).

Results: In 2016, 46 women and 47 men aged 65 to 94 years were admitted to the psychiatric wards (two women and two men were admitted twice). A total of 12 falls involving nine women and two men were reported. Over $66 \%$ of falls involved patients with dementia, $75 \%$ involved women, and over $50 \%$ occurred on the way to the bathroom, mostly during the night shift when the staffing level was low. In the pilot study, of 30 consecutive psychogeriatric inpatients, 20 were classified as high risk by the WSFRAT and 10 of them were also classified as high risk by the MFS. Those classified as high risk by WSFRAT matched perfectly with those classified as high risk by MFAC. However, $30 \%$ of those classified as high risk by MFAC were not classified as high risk by MFS.

Conclusions: Patients with dementia and women had higher risk of fall. Extra proportion of at-risk patients are able to benefit from additional fall risk interventions when WSFRAT is used instead of MFS. Further studies are needed to calculate the psychometric properties of WSFRAT.
\end{abstract}

Key words: Accidental falls; Geriatric psychiatry

MMC Wong, MBBS, MRCPsych, FHKC Psych, FHKAM (Psychiatry), Department of Psychiatry, United Christian Hospital, Hong Kong PF Pang, MBChB, MRCPsych, FHKC Psych, FHKAM (Psychiatry), Department of Psychiatry, United Christian Hospital, Hong Kong

Address for correspondence: $\operatorname{Dr} M M C$ Wong, Department of Psychiatry, United Christian Hospital, Hong Kong.Email: wmc009@ha.org.hk

Submitted: 19 December 2017; Accepted: 21 June 2018

\section{Introduction}

Falls among elderly inpatients is common and can be associated with severe morbidity, functional decline, and mortality. ${ }^{1}$ Patients at a psychiatric unit are at greater fall risk owing to a multiplicity of issues including psychotropic medications, over-activity, and confusion. ${ }^{2,3}$ The incidence of falls in psychiatric units is higher than that in general acute care hospital units (13.1-25 vs 3-5 per 1000 inpatientdays). ${ }^{2}$ In a study in Western Australia comparing two psychiatric units, 139 falls were reported in a 12-month period, and the incidence of falls per 1000 bed-days was higher in one psychiatric unit than in any other clinical specialty unit of the tertiary hospital. ${ }^{4}$ Unlike those in the medical ward, older people in psychiatric units may be restless and agitated and spend less time on their beds. They may experience dizziness, and at risk of falling when getting up from chairs, beds and particularly in bathrooms, owing to old age and comorbidities. ${ }^{2}$

There are limited studies on falls in psychogeriatric inpatients despite the high number of falls. ${ }^{5}$ Many hospitals use a single instrument that has not been validated in psychiatric settings for assessment of fall risk in all units. ${ }^{6}$ The Morse Fall Scale (MFS) is a fall assessment tool for patients in acute medical-surgical units, but it has not been validated in psychiatric inpatients. Toileting is an important factor contributing to falling, which the MFS does not capture. $^{?}$

Despite adoption of the fall risk protocol, the number of falls per 1000 patient-days in a geriatric psychiatric facility remained at 4.83 , which was higher than that in the rest of the hospital units. ${ }^{8}$ Psychiatric inpatients have unique risk factors for fall that are compounded by other factors such as medications, anxiety, and agitation. A fall risk assessment tool for psychiatric inpatients is therefore needed. The Wilson Sims scale and the Edmonson 
Psychiatric Fall Risk Assessment Tool are recommended fall risk assessment tools for psychiatric adult and geriatric patients. ${ }^{9,10}$ A major difference between these two scales is the inclusion of nurse judgment in the Wilson Sims scale. A clinical judgment of an experienced professional is as good as a validated fall prediction tool. ${ }^{11}$

United Christian Hospital (UCH) is an acute general hospital in Hong Kong. It serves a population of about 1.1 million of which 150000 were aged $\geq 65$ years. It has two psychiatric wards with a total of 80 beds (16 of which for patients aged $\geq 65$ years); each ward has five cubicles. One cubicle with eight beds is for female elderly patients and another with eight beds is for male elderly patients. These patients share the same day room with male adult patients. Each ward has four nursing staff and one to two health care assistants on duty in the day shift, and two nursing staff and one health care assistant on duty in the night shift.

In 2016, among all psychiatric inpatients, 25 falls occurred, 12 of which involved elderly patients. In 2015, 25 falls occurred but only 9 of which involved elderly patients. The rate of fall per 1000 bed-day increased from $1.54 \%$ in 2015 to $2.05 \%$ in 2016. In UCH, the MFS is used to assess the risk of fall in all inpatients, and fall prevention and management guideline is followed in the psychiatric wards. ${ }^{12}$ All fall incidents are reported through the Advance Incident Reporting System to the governing authority and follow-up actions are taken promptly. A fall prevention working group (involving psychiatric doctors, nurses, physiotherapist, and occupational therapist) reviews all fall cases and discuss measures to prevent fall in the psychiatric ward. Those deemed to be at high fall risk are referred to the Fall Prevention and Intervention Programme for provision of suitable walking aids and training. Patients and their carers are educated on fall risk and precaution. Special nursing precautions include putting on a fall risk signage, use of bed exit alarm, provision of an easily accessible call bell, use of bed rail, and sitting arrangement in close proximity to nurse station.

This study aimed to investigate factors associated with falls in psychogeriatric inpatients and to compare two fall risk assessment tools for psychiatric patients.

\section{Methods}

This study had two parts. First, all fall reports involving psychogeriatric inpatients in UCH in 2016 were retrieved from the Advance Incident Reporting System and retrospectively reviewed. Factors associated with the falls were identified by comparing patients who had a fall incident and those who did not for development of a fall prevention programme.

Second, in a pilot study, 30 consecutive psychogeriatric inpatients were assessed for fall risk using the Morse Fall Scale (MFS) and the Wilson Sims Fall Risk Assessment Tool (WSFRAT). The WSFRAT included clinical judgment by nursing staff so that they could upgrade the fall risk of a patient. Those classified as high risk by both scales were compared with those classified as high risk by the modified Functional Ambulation Classification (MFAC).${ }^{13}$ MFAC was developed by the Coordinating Committee in Physiotherapy of the Hospital Authority and is used by physiotherapists to assess walking capacity of psychogeriatric patients. MFAC scores are from 1 to 7 on a 7-point Likert scale. Patients with a MFAC score of 1 to 5 were classified as high risk (with abnormalities of balance and gait) and those with a score of 6 to 7 were classified as low risk.

Statistical analysis was performed using SPSS (Windows version 21.0, SPSS, Chicago [IL], US). Comparisons of patients with and without falls were made using the $t$ test for continuous variables and the Chi-squared test or Fisher's exact test for categorical variables.

\section{Results}

In 2016, 46 women and 47 men aged 65 to 94 years (mean \pm standard deviation $[\mathrm{SD}], 75.1 \pm 7.3$ years) were admitted to the psychiatric wards (two women and two men were admitted twice). Of the 93 patients, 35 were diagnosed with mood-related problems (including adjustment disorder, depressive episode, mixed anxiety and depressive disorder, and generalized anxiety disorder), 25 with dementia, 19 with psychotic disorder (including schizophrenia, delusional disorder, and schizoaffective disorder), four with bipolar affective disorder, two with delirium, one with alcoholrelated disorder, and seven with two psychiatric diagnoses (one of them being dementia) [Table 1].

Table 1. Diagnoses of psychogeriatric inpatients admitted in 2016

\begin{tabular}{lc}
\hline Diagnosis & $\begin{array}{c}\text { No. of patients } \\
(\mathbf{n}=\mathbf{9 3})\end{array}$ \\
Depression & 19 \\
\hline Adjustment disorder & 9 \\
\hline Generalised anxiety disorder & 3 \\
\hline Mixed anxiety and depressive disorder & 4 \\
\hline Dementia & 25 \\
\hline Schizophrenia & 11 \\
\hline Delusional disorder & 7 \\
\hline Bipolar affective disorder & 4 \\
\hline Schizoaffective disorder & 1 \\
\hline Alcohol-related disorder & 1 \\
\hline Delirium & 2 \\
\hline Dementia \& schizophrenia & 2 \\
\hline $\begin{array}{l}\text { Dementia \& mixed anxiety and } \\
\text { depressive disorder }\end{array}$ & 2 \\
\hline Dementia \& bipolar affective disorder & 2 \\
\hline Dementia \& alcohol-related disorder & 1 \\
\hline
\end{tabular}


A total of 12 falls involving nine women and two men aged 66 to 94 years (mean $\pm \mathrm{SD}, 78.4 \pm 9$ years) were reported. Of the 11 patients, seven were diagnosed with dementia (two of them also had either schizophrenia or mixed anxiety and depressive disorder), three with depression, and one with schizophrenia. Compared with those who did not fall, those who had a fall incident were more likely to be women $(81.8 \%$ vs $45.1 \%, p=0.027)$ and have dementia $(63.6 \%$ vs $43.9 \%, \mathrm{p}=0.04)$ but their age was similar $(\mathrm{t}$-value $=1.6, \mathrm{p}=0.1)$.

According to the MFS, before their fall incidents, four patients were classified as high risk, six as medium risk, and one as low risk. One of them was classified as medium risk and then as high risk after his first fall.

According to the Advance Incident Reporting System, seven falls were related to toileting (all involved women) in early morning ( $\mathrm{n}=4 ; 3$ of them fell near bedside), before midnight $(\mathrm{n}=1)$, in the afternoon $(\mathrm{n}=1)$, and in the evening $(n=1)$. For the remaining five falls, one woman with Parkinson's disease and dementia rolled from her bed; one woman fell when she rushed back for lunch; two patients lost balance without specific precipitating factors; and one of these two patients fell again when he was pulled down by an adolescent in the same ward who was annoyed by his behavioural and psychological symptoms of dementia. Of the 12 falls, eight did not result in any injury and four resulted in minor injuries such as loss of a tooth $(\mathrm{n}=1)$, bodily pain $(n=2)$, and superficial abrasion over the left forehead $(n=1)$.

No fall occurred within the first week of hospitalisation. No patient had a drop in postural blood pressure immediately after the fall. Seven patients had upward titration of psychotropic drugs within 5 days of the fall. The 11 patients were taking a mean number of psychotropic drugs of 3.1 (range, 1-5) and had a mean of 2.3 (range, 1-4) comorbidities (diabetes mellitus, hypertension, Parkinson's disease, osteoarthritis of the knees, and congestive heart failure). Those who had a fall incident and those who did not were comparable in terms of the number of psychotropic drugs taken and the number of comorbidities.

In the pilot study, of 30 consecutive psychogeriatric inpatients, 17 were classified as high risk by the WSFRAT and 8 of them were also classified as high risk by the MFS (Table 2) During the pilot study, two of the 8 patients

Table 2. Fall risk of patients classified by the Morse Fall Scale and the Wilson Sims Fall Risk Assessment Tool (WSFRAT)

\begin{tabular}{|lc|}
\hline Fall risk & $\begin{array}{c}\text { No. }(\%) \text { of } \\
\text { patients }(\mathbf{n}=\mathbf{3 0})\end{array}$ \\
\hline High risk by both scales & $8(26.67)$ \\
\hline Low or medium risk by both scales & $13(43.3)$ \\
\hline High risk by WSFRAT only & $9(30)$ \\
\hline
\end{tabular}

who were classified as high risk by both scales had a fall incident; sensitivity was $100 \%$ when the risk was dichotomised to high risk or not. Those classified as high risk by WSFRAT matched perfectly with those classified as high risk by MFAC. However, $30 \%$ of those classified as high risk by MFAC were not classified as high risk by MFS.

\section{Discussion}

The National Institute for Health and Clinical Excellence guidance no longer recommends the use of fall risk assessment tools ${ }^{14}$ as most falls occur in patients classified as low risk. Instead, it recommends that all older individuals should be considered to be at high risk of falls. Nonetheless, in view of limited manpower, it is more desirable to use a sensitive fall risk assessment tool to detect those with high fall risk and refer them to the Fall Prevention and Intervention Programme. Our frontline nursing staff have been educated about the constant change in risk factor of inpatients and will reassess the fall risk whenever needed. We decided to use the WSFRAT as it allows clinical judgment of nurses when interpreting fall risk. After the pilot study, WSFRAT was used together with MFS in all psychogeriatric patients, and an extra $30 \%$ of at-risk patients could benefit from fall risk interventions. The pilot study would extend to patients aged 60 to 64 years to determine whether the WSFRAT can be used routinely for all psychiatric inpatients.

Although the MFAC may substitute the WSFRAT in psychogeriatric patients, MFAC score cannot be available immediately upon admission and does not cover risk factors (such as use of psychotropics and undergoing detoxification) that are specific to psychiatric inpatients. MFAC only assesses mobility, but most adult patients do not have mobility problem (unlike psychogeriatric patients).

In our study, over half of the patients fell on the way to the bathroom. This finding was compatible with that in a study reporting that $>45 \%$ of inpatient falls were associated with elimination-related activities. ${ }^{7}$ Patients deemed to have fall risk were advised to seek help before they left their bed or chair, but they went to the bathroom without calling for assistance. Five of these seven falls occurred during the night shift when the staffing level was low, which is associated with a higher fall risk..$^{15}$ In three of these falls, the patients was found next to the bedside, indicating that they fell shortly after they tried to get up. Nonetheless, use of restraint during sleep was avoided, as it is associated with an even higher fall risk and increased severity of injuries among elderly inpatient fallers. ${ }^{16}$ Removal of restraints does not increase fall risk or subsequent injuries from falls in nursing home residents. ${ }^{17}$ The use of bed-exit alarm can accurately detect bed absence episodes among geriatric inpatients and alert nurses accordingly, and significantly reduces the total workload score, with high acceptability among the nurses. ${ }^{18}$ Use of bed exit alarm is encouraged for suitable psychogeriatric female patients at night time to alert staff when they attempted to leave their bed without 
calling for assistance from staff. It is being used in two patients with dementia at night.

Although some hospitals use diapers at night for patients with dementia, this might not reduce the frequency of toilet visits, as patients are not used to urinating into diapers and insist on getting up to urinate in the toilet. Although patients with dementia can be potted at fixed intervals (ie, timed voiding) and prompted by nurses to void (ie, prompted voiding) at night, these are more labour intensive than diaper use and are practically difficult during night shift. Male inpatients did not have fall incidents related to toileting, probably because of the use of bed pans or urinals. In a multifaceted fall prevention programme, the use of bedside commode is recommended to increase accessibility and efficiency in patient care delivery. ${ }^{19}$ Nonetheless, use of commode chair is limited by the lack of space in the psychogeriatric cubicle. Use of bed pan is encouraged for suitable female patients to reduce the travelling distance to the toilet.

Some falls occurred few days after upward titration of psychiatric medications. It is not uncommon for psychogeriatric inpatients to be put on multiple psychiatric medications. The likelihood of falling increases with the use of neuroleptic and antipsychotic drugs. ${ }^{20}$ Combination of psychotropics or other drugs might increase sedating effect and is prone to drug interactions. Fall risk is associated with polypharmacy when at least one fall risk-increasing drug is part of the daily regimen. ${ }^{21}$ These older patients commonly have comorbidities that further increase the number of medications. A slow titration speed and frequent monitoring of side-effects are important for psychogeriatric patients. Older people on psychotropic medications should have their medication reviewed and, if necessary, discontinued to reduce the risk of falling. ${ }^{14}$ Psychogeriatric doctors should be aware of the risk of fall in psychogeriatric patients and be cautious in prescribing medications to reduce iatrogenic cause of fall.

The mixed ward setting may also increase the fall risk of elderly patients. Fall incidents related to elderly patients being pushed down by teenage patients were recorded. It is hoped that this problem could be solved after redevelopment of the hospital. Fortunately, no falls resulted in major injury or fracture. Over $75 \%$ of falls involving older adult psychiatric inpatients resulted in no injury. ${ }^{22}$ This may be due to better physical condition of elderly patients in the psychiatric ward than those in medical wards.

Staff supervision is important. Adequate staff availability when residents exit bed is necessary. ${ }^{23}$ Close supervision is important in fall prevention in ambulant patients; this includes checks at least every hour when patients are resting. ${ }^{24} \mathrm{~A}$ higher staff-to-patient ratio and direct monitoring of the two psychogeriatric cubicles by designated staff especially during the night shift are necessary to prevent falls. There will be an addition of two staff for the two psychiatric wards during night shift. Staff working with older people at risk of falls should update their knowledge and skills regarding causes and prevention of falls. ${ }^{5}$ Training on fall prevention and transfer of patients is provided to ward staff regularly.

In this study, diagnoses were made by the case doctor and endorsed by the associate consultant or consultant of the psychogeriatric team in the multidisciplinary ward round. Cognitive assessment was performed for all patients. Psychiatric diagnoses including dementia were made accurately and comprehensively. However, the numbers of patients and fall cases were small. Circumstances related to the fall were based on the Advance Incident Reporting System only. Documentation of fall incidents is adequate and relevant, as the fall prevention working group regularly provides feedbacks to ward nurses. Findings of this pilot study can be used to guide planning of further multicentre studies with larger sample size so that psychometric properties of WSFRAT can be calculated.

\section{Conclusions}

The majority of falls involved patients with dementia, women, or patients on the way to the bathroom, mostly during the night shift when the staffing level was low. Use of bed exit alarms and bed pans is encouraged for suitable patients. Extra proportion of at-risk patients are able to benefit from additional fall risk interventions when WSFRAT is used instead of MFS. Psychogeriatric doctors should be aware of drug titration speed and side-effects of medications. A higher staff-to-patient ratio should be in place for prevention of falls in ambulant psychogeriatric patients.

\section{Acknowledgement}

We would like to thank all team members who have assisted in the pilot study of the fall rating scale.

\section{Declaration}

No financial support is received. The authors have no conflict of interest to disclose.

\section{References}

1. Oliver D, Britton M, Seed P, Martin FC, Hopper AH. Development and evaluation of evidence based risk assessment tool (STRATIFY) to predict which elderly inpatients will fall: case-control and cohort studies. BMJ 1997;315:1049-53. Crossref

2. Blair E, Gruman C. Fall in an inpatient geriatric psychiatric population. J Am Psychiatr Nurses Assoc 2005;11:351-4. Crossref

3. Finkelstein $\mathrm{E}$, Prabhu $\mathrm{M}$, Chen $\mathrm{H}$. Increased prevalence of falls among elderly individuals with mental health and substance abuse conditions. Am J Geriatr Psychiatry 2007;15:611-9. Crossref

4. Heslop K, Wynaden D, Bramanis K, Connolly C, Gee T, Griffiths R, et al. Assessing falls risk in older adult mental health patients: a Western Australian review. Int J Ment Health Nurs 2012;21:567-75. Crossref

5. Bunn F, Dickinson A, Simpson C, Narayanan V, Humphrey D, Griffiths C, et al. Preventing falls among older people with mental health problems: a systematic review. BMC Nurs 2014;13:4. Crossref

6. Rutledge D, Donaldson N, Pravikoff D. Update 2003: Fall risk assessment and prevention in hospitalized patients. Online J Clin 
Innovations 2003;6:1-55.

7. Tzeng HM. Understanding the prevalence of inpatient falls associated with toileting in adult acute care settings. J Nurs Care Qual 2010;25:2230. Crossref

8. Knight M, Coakley C. Fall risk in patients with acute psychosis. J Nurs Care Qual 2010;25:208-15. Crossref

9. Edmonson D, Robinson S, Hughes L. Development of the Edmonson Psychiatric Fall Risk Assessment Tool. J Psychosoc Nurs Ment Health Serv 2011;49:29-36. Crossref

10. Abraham S. Looking for a psychiatric fall risk assessment tool. Ann Psychiatry Ment Health 2016;4:1061.

11. Wilson SC, Fettes S, Sims K. Gravity: It's not a suggestion-It's the law! The development of the Wilson Sims Psychiatric Fall Risk Assessment. J Am Psychiatr Nurses Assoc 2014;20:83.

12. Quality and Safety Committee of Department of Psychiatry. Standard Operating Procedures on Fall Prevention and Management in Wards of the Department of Psychiatry, United Christian Hospital, Hong Kong. Unpublished data, United Christian Hospital; 2016.

13. Coordinating Committee in Physiotherapy Hospital Authority. Validation study of modified Rivermead Mobility Index and modified Functional Ambulation Classification for stroke patients. Unpublished data, Hospital Authority; 2007.

14. National Institute for Health and Clinical Excellence. Falls: assessment and prevention of falls in older people (CG 161). Manchester: NICE; 2013.

15. Staggs VS, Knight JE, Dunton N. Understanding unassisted falls: effects of nurse staffing level and nursing staff characteristics. J Nurs Care Qual 2012;27:194-9. Crossref
16. Tan KM, Austin B, Shaughnassy M, Higgins C, McDonald M, Mulkerrin EC, et al. Falls in an acute hospital and their relationship to restraint use. Ir J Med Sci 2005;174:28-31. Crossref

17. Capezuti E, Strumpf NE, Evans LK, Grisso JA, Maislin G. The relationship between physical restraint removal and falls and injuries among nursing home residents. J Gerontol A Biol Sci Med Sci 1998;53:M47-52. Crossref

18. Subermaniam K, Welfred R, Subramanian P, Chinna K, Ibrahim F, Mohktar MS, et al. The effectiveness of a wireless modular bed absence sensor device for fall prevention among older inpatients. Front Public Health 2017;4:292. Crossref

19. Tzeng HM. A feasibility study of providing folding commode chairs in patient bathrooms to reduce toileting-related falls in an adult acute medical-surgical unit. J Nurs Care Qual 2011;26:61-8. Crossref

20. Woolcott JC, Richardson KJ, Wiens MO, Patel B, Marin J, Khan KM, et al. Meta-analysis of the impact of 9 medication classes on falls in elderly persons. Arch Intern Med 2009;169:1952-60. Crossref

21. Ziere G, Dieleman JP, Hofman A, Pols HA, van der Cammen TJ, Stricker BH. Polypharmacy and falls in the middle age and elderly population. Br J Clin Pharmacol 2006;61:218-23. Crossref

22. Stubbs B. Falls in older adult psychiatric inpatients. Int Psychogeriatr 2010;22:160. Crossref

23. Capezuti E, Talerico KA, Cochran I, Becker H, Strumpf N, Evans L. Individualized interventions to prevent bed-related falls and reduce siderail use. J.Gerontol Nurs 1999;25:26-34. Crossref

24. McMinn B, Booth A, Grist E, O'Brien A. Falls and fall injury in mental health inpatient units for older people. Aust Nurs Midwifery $\mathbf{J}$ 2016;24:26-9. 